\title{
Achievable region of reliabilities in multiple hypotheses two-stage testing for the pair of families of distributions
}

\author{
Farshin Hormozi-Nejad \\ Department of Mathematics and Statistics, Ahvaz Branch, Islamic Azad University, Ahvaz, Iran \\ Email: hormozi-nejad@iauahvaz.ac.ir
}

Copyright (C2014 Farshin Hormozi-Nejad. This is an open access article distributed under the Creative Commons Attribution License, which permits unrestricted use, distribution, and reproduction in any medium, provided the original work is properly cited.

\begin{abstract}
The achievable region of reliabilities in the model with several possible hypothetical probability distributions partitioned into the pair of families is considered. The achievable region for many hypotheses testing was examined by Tuncel. Decisions concerning realized probability distribution of the object must be made on the base of the samples which are received in each stage of the two-stage test. It is proved that the defined region for the vectors of reliabilities in the two-stage test characterizes the set of all achievable vectors and advantages of the two-stage testing are revealed.
\end{abstract}

Keywords: LAO test, method of types, multiple hypotheses testing, reliability, two-stage test.

\section{Introduction}

In present paper, the approach of Tuncel [11] for the achievable region of reliabilities of testing of the model that follows a probability distribution (PD) from one of a pair of families is developed. The list of $S$ hypothetical PDs is given. The statistical problem is to detect actual PD from this list and to find the achievable region using a sample of $N$ experiments outcomes. The overwhelming majority of published works are dedicated to the case of two hypotheses [10]. The Logarithmically asymptotically optimal (LAO) testing of two hypotheses is the procedure ensuring the best exponential decrease with growing $N$ of the error probability of one hypothesis subject to the given exponent of the error probability of the other hypotheses. Hoeffding [9] and later Tusnady [12] and others investigated LAO tests in the case of two hypotheses. The LAO testing for multiple hypotheses first was considered by Haroutunian [6]. In $[1,5,6]$ some results on multiple hypotheses testing and identification are presented. The two-stage tests have become popular in applications, especially in the field of clinical trials and genomics [5]. Ahlswede and Haroutunian [1] proposed the problem of statistical hypotheses optimal testing and identification for many objects. Haroutunian et al. [7] investigated reliability criteria in information theory and in statistical hypothesis testing. Yessayan et al. [13] solved achievable region of reliabilities in testing of many hypotheses for two independent objects. The model of the two-stage LAO testing of multiple hypotheses for the pair of families of distributions is investigated in [8].

The map of this correspondence is as follows. In Section 2, briefly the problem statement and some needed definitions are declared. In Section 3, achievable region in the one-stage test considered by E. Tuncel [11] is introduced. In Section 4, the analysis of achievable region at the first stage of two-stage test is investigated. In Section 5, achievable region at the second stage of two-stage test is studied. In Section 6, achievable region at the 
two-stage test composed of the first and the second stages of test is declared. Finally in last Section, a conclusion for achievable region at two-stage test in the pair of families of PDs is disclosed.

\section{Problem statement and definitions}

Let $X$ be a random variable $(\mathrm{RV})$ taking values in the finite set $\mathcal{X}$ and $\mathcal{P}(\mathcal{X})$ be the space of all possible PDs on $\mathcal{X}$. Suppose $S$ known hypothetical PDs from $\mathcal{P}(\mathcal{X})$ are given and they are partitioned into two disjoint families of PDs. The first family includes $R$ PDs $P_{s}=\left\{P_{s}(x), x \in \mathcal{X}\right\}, s=\overline{1, R}$, and the second family includes $S-R$ PDs $P_{s}=\left\{P_{s}(x), x \in \mathcal{X}\right\}, s=\overline{R+1, S}$ such that the considered object characterized by RV $X$ follows to one of this $S$ PDs.

Let $\mathrm{x}=\left(x_{1}, x_{2}, \ldots, x_{N}\right)$ be a vector of results of $N$ independent observations of the RV $X$ and $N=N_{1}+N_{2}$, such that:

$$
\begin{gathered}
N_{1}=\lceil\psi N\rceil, \quad N_{2}=[(1-\psi) N], \quad 0<\psi<1, \\
\mathrm{x}_{1}=\left(x_{1}, x_{2}, \ldots, x_{N_{1}}\right), \quad \mathrm{x}_{1} \in \mathcal{X}^{N_{1}}, \\
\mathrm{x}_{2}=\left(x_{N_{1}+1}, x_{N_{1}+2}, \ldots, x_{N}\right), \quad \mathrm{x}_{2} \in \mathcal{X}^{N_{2}}, \\
\mathrm{x}=\left(\mathrm{x}_{1}, \mathrm{x}_{2}\right), \mathrm{x} \in \mathcal{X}^{N}, \mathcal{X}^{N}=\mathcal{X}^{N_{1}} \times \mathcal{X}^{N_{2}} .
\end{gathered}
$$

An achievable region of the problem of many hypotheses two-stage testing concerning an object at two stages of test is investigated. At first stage of test, one family of PDs is denoted, then at the next stage, object's distribution between selected family of PDs is detected.

The statistician must accept one of the families of PDs in the first stage on the base of a sample $\mathrm{x}_{1}$ and then he accept one of the PDs in the second stage, on the base of other sample $\mathrm{x}_{2}$.

The procedure of making decision on the base of $N$ observations called the test and denote it by $\phi^{N}$ for the one-stage test and by $\Phi^{N}$ for the two-stage test. The test $\Phi^{N}$ may be composed of the pair of tests $\varphi_{1}^{N_{1}}$ and $\varphi_{2}^{N_{2}}$ for two consecutive stages and it is written by $\Phi^{N}=\left(\varphi_{1}^{N_{1}}, \varphi_{2}^{N_{2}}\right)$. The first stage of making decision for selecting a family of PDs is a non-randomized test $\varphi_{1}^{N_{1}}\left(\mathrm{x}_{1}\right)$. The next stage of making decision for PD acceptance in the determined family of PDs is a non-randomized test $\varphi_{2}^{N_{2}}\left(\mathrm{x}_{2} \mid \varphi_{1}^{N_{1}}\left(\mathrm{x}_{1}\right)=i\right), i=1,2$, on the base of the sample $\mathrm{x}_{2}$ and on the result 1 or 2 of the test $\varphi_{1}^{N_{1}}\left(\mathrm{x}_{1}\right)$.

We need some formulations of method of types. Let $N(x \mid \mathrm{x})$ be the number of repetitions of the element $x \in \mathcal{X}$ in the vector $\mathrm{x} \in \mathcal{X}^{N}$. The distribution

$$
Q_{\mathrm{x}}(x) \triangleq \frac{N(x \mid \mathrm{x})}{N}, \quad x \in \mathcal{X},
$$

is called in information theory, "the type" of $x[1,3,4]$.

Let $\mathcal{P}^{N}(\mathcal{X})$ be the set of all possible types of vectors from $\mathcal{X}^{N}$ and $\mathcal{T}_{Q}^{N}$ be type class of PD $Q$, the set of all vectors x of the type $Q \in \mathcal{P}^{N}(\mathcal{X})$ [3]. The divergence (Kullback-Leibler distance) for PDs $P$ and $Q$, is defined as follows $[3,4,6]$ :

$$
\mathrm{D}(Q \| P) \triangleq \sum_{x \in \mathcal{X}} Q(x) \log \frac{Q(x)}{P(x)}
$$

The number of distinct types for vectors of length $N$ grows at most polynomially with $N[2,3]$. More specifically quantity of elements of $\mathcal{P}^{N}(\mathcal{X})$ can be estimated as follows

$$
\left|\mathcal{P}^{N}(\mathcal{X})\right| \leq(N+1)^{|\mathcal{X}|} .
$$

For any type $Q$, population of the type class $\mathcal{T}_{Q}^{N}$ is estimated as follows $[2,3]$

$$
(N+1)^{-|\mathcal{X}|} \exp \{N \mathrm{H}(Q)\} \leq\left|\mathcal{T}_{Q}^{N}\right| \leq \exp \{N \mathrm{H}(Q)\},
$$

where $\mathrm{H}(Q)$ denotes the entropy of distribution $Q[2,3]$

$$
\mathrm{H}(Q) \triangleq-\sum_{x \in \mathcal{X}} Q(x) \log Q(x) .
$$

Probability of members $\mathrm{x}$ of a type class $\mathcal{T}_{Q}^{N}$ provided $\mathrm{x}$ is i.i.d. by $\mathrm{PD} P$ is given by the formula $[2,3]$

$$
\mathrm{P}^{N}(\mathrm{x})=\exp \{-N[\mathrm{H}(Q)+\mathrm{D}(Q \| P)]\} .
$$

Bounds on the total probability of a type class $\mathcal{T}_{Q}^{N}$ when $\mathrm{x}$ is i.i.d. by PD $P$ are $[2,3]$

$$
(N+1)^{-|\mathcal{X}|} \exp \{-N \mathrm{D}(Q \| P)\} \leq \mathrm{P}^{N}\left(\mathcal{T}_{Q}^{N}\right) \leq \exp \{-N \mathrm{D}(Q \| P)\}
$$




\section{Achievable region at the one-stage test}

In multiple hypotheses testing, decision making on the base of $N$-sample x for accepting a realized PD between $S$ PDs $P_{s}, s=\overline{1, S}$, the test $\phi^{N}$ can be defined by partitioning the sample space $\mathcal{X}^{N}$ into $S$ disjoint subsets $\mathcal{G}_{s}^{(N)}, s=\overline{1, S}$. The set $\mathcal{G}_{s}^{(N)}$ consists of all vectors x for which $s$-th PD is adopted.

$$
\mathcal{G}_{s}^{(N)} \triangleq\left\{\mathrm{x}: \phi^{N}(\mathrm{x})=s\right\}, \quad s=\overline{1, S}, \quad \bigcup_{s=1}^{S} \mathcal{G}_{s}^{(N)}=\mathcal{X}^{N} .
$$

Let $\alpha_{l \mid k}$ be the probability of the erroneous acceptance of PD $P_{l}$ provided $P_{k}$ is true

$$
\alpha_{l \mid k}\left(\phi^{N}\right) \triangleq P_{k}^{N}\left(\mathcal{G}_{l}^{(N)}\right), \quad l, k=\overline{1, S}, \quad l \neq k .
$$

The probability to reject $P_{k}$, when it is true, is

$$
\alpha_{k \mid k}\left(\phi^{N}\right) \triangleq P_{k}^{N}\left(\overline{\mathcal{G}}_{k}^{(N)}\right)=\sum_{l \neq k} \alpha_{l \mid k}\left(\phi^{N}\right), \quad l, k=\overline{1, S} .
$$

The infinite sequences of tests is denoted by $\phi$, corresponding the reliabilities of $\phi$ are defined as follows

$$
E_{l \mid k}(\phi) \triangleq \liminf _{N \rightarrow \infty}\left\{-\frac{1}{N} \log \alpha_{l \mid k}\left(\phi^{N}\right)\right\}, l, k=\overline{1, S} .
$$

We now formally define the concept of achievability of a set of error probability exponents in $S$-ary hypotheses testing. Consider the $S(S-1)$-dimensional Euclidean space, and index the dimensions as $\{l, k\}$, where $l, k=$ $\overline{1, S}$. We denote by $\mathbf{E}=\left\{E_{l \mid k}, l \neq k\right\}$ the vector, element of which correspond to the set of error exponents $-\frac{1}{N} \log \left\{P_{k}^{N}\left(\mathcal{G}_{l}^{(N)}\right)\right\}$.

Definition 3.1 [11] The set of error exponents indicated by the vector $\mathbf{E}$ is called achievable if for all $\varepsilon>0$ and large enough $N$ there exists a decision scheme $\mathcal{G}_{s}^{(N)}, s=\overline{1, S}$, satisfying for $l, k=\overline{1, S}, l \neq k$ the following conditions:

$$
-\frac{1}{N} \log \alpha_{l \mid k}\left(\phi^{N}\right)>E_{l \mid k}(\phi)-\varepsilon .
$$

The set of all achievable vectors is defined by $\mathcal{R}$.

Let us define a region $\mathcal{E}$ in the error-exponent space as follows:

$$
\mathcal{E} \triangleq\left\{\mathbf{E}: \forall Q, \exists l, \mathrm{D}\left(Q \| P_{k}\right)>E_{l \mid k}(\phi), \quad k \neq l, \quad l, k=\overline{1, S}\right\} .
$$

Theorem 3.2 [11] The following inclusion take place $\mathcal{E} \subset \mathcal{R}$. Conversely if $\mathbf{E} \in \mathcal{R}$, then for any $\delta>0, \mathbf{E}_{\delta} \in \mathcal{E}$, where $\mathbf{E}_{\delta}=\left\{E_{l \mid k}(\phi)-\delta\right\}$.

\section{Achievable region at the first stage of two-stage test}

Suppose $\mathcal{D}_{1}=\{\overline{1, R}\}$ and $\mathcal{D}_{2}=\{\overline{R+1, S}\}$ and the pair of disjoint families of PDs $\mathcal{P}_{1}$ and $\mathcal{P}_{2}$ :

$$
\mathcal{P}_{1}=\left\{P_{s}, \quad s \in \mathcal{D}_{1}\right\}, \quad \mathcal{P}_{2}=\left\{P_{s}, \quad s \in \mathcal{D}_{2}\right\} .
$$

The first stage of decision making for selection of a family of PDs is a test $\varphi_{1}^{N_{1}}\left(\mathrm{x}_{1}\right)$, which can be defined by partitioning the sample space $\mathcal{X}^{N_{1}}$ into the pair of disjoint subsets

$$
\mathcal{A}_{i}^{N_{1}} \triangleq\left\{\mathrm{x}_{1}: \varphi_{1}^{N_{1}}\left(\mathrm{x}_{1}\right)=i\right\}, \quad i=1,2, \quad \mathcal{A}_{1}^{N_{1}} \cup \mathcal{A}_{2}^{N_{1}}=\mathcal{X}^{N_{1}} .
$$

The set $\mathcal{A}_{i}^{N_{1}}$ consists of all vectors $\mathrm{x}_{1}$ for which $i$-th family of PDs is adopted. 
The test $\varphi_{1}^{N_{1}}\left(\mathrm{x}_{1}\right)$ have two kinds of errors for the pair of hypotheses $P \in \mathcal{P}_{i}, i=1,2$. Let $\alpha_{2 \mid 1}^{\prime}\left(\varphi_{1}^{N_{1}}\right)$ be the probability of the erroneous acceptance of the second family of PDs provided the first family of PDs is true. And $\alpha_{1 \mid 2}^{\prime}\left(\varphi_{1}^{N_{1}}\right)$ be the probability of the erroneous acceptance of the first family of PDs provided the second family of PDs is true:

$$
\begin{aligned}
& \alpha_{2 \mid 1}^{\prime}\left(\varphi_{1}^{N_{1}}\right) \triangleq \max _{s: s \in \mathcal{D}_{1}} P_{s}^{N_{1}}\left(\mathcal{A}_{2}^{N_{1}}\right), \\
& \alpha_{1 \mid 2}^{\prime}\left(\varphi_{1}^{N_{1}}\right) \triangleq \max _{s: s \in \mathcal{D}_{2}} P_{s}^{N_{1}}\left(\mathcal{A}_{1}^{N_{1}}\right) .
\end{aligned}
$$

And corresponding reliabilities of the infinite sequence of tests $\varphi_{1}$, are:

$$
E_{i \mid j}^{\prime}\left(\varphi_{1}\right) \triangleq \liminf _{N_{1} \rightarrow \infty}\left\{-\frac{1}{N_{1}} \log \alpha_{i \mid j}^{\prime}\left(\varphi_{1}^{N_{1}}\right)\right\}, \quad i, j=1,2
$$

We denote by $\mathbf{E}^{\prime}=\left\{E_{i \mid j}^{\prime}, i \neq j, i, j=1,2\right\}$ the vector, element of which correspond to the set of error exponents $-\frac{1}{N_{1}} \log \left\{\alpha_{i \mid j}^{\prime}\left(\varphi_{1}^{N_{1}}\right)\right\}$.

For given $E_{2 \mid 1}^{\prime}$, the test $\varphi_{1}^{N_{1}}$ is defined by partitioning $\mathcal{X}^{N_{1}}$ into

$$
\mathcal{A}_{1}^{\left(N_{1}\right)}=\bigcup_{Q_{\mathbf{x}_{1}}: \min _{s: s \in \mathcal{D}_{1}} \mathrm{D}\left(Q_{\mathbf{x}_{1}} \| P_{s}\right) \leq E_{2 \mid 1}^{\prime}} \mathcal{T}_{Q_{\mathbf{x}_{1}}}^{N_{1}}
$$

and $\mathcal{A}_{2}^{\left(N_{1}\right)}=\mathcal{X}^{N_{1}} \backslash \mathcal{A}_{1}^{\left(N_{1}\right)}$ disjoint subsets [8].

Definition 4.1 [11] The set of error exponents indicated by vector $\mathbf{E}^{\prime}$ is called achievable if for all $\varepsilon>0$ and large enough $N_{1}$ there exists a decision schemes $\mathcal{A}_{i}^{\left(N_{1}\right)}, i=1,2$ satisfying for $i, j=1,2, i \neq j$ the following conditions:

$$
-\frac{1}{N_{1}} \log \alpha_{i \mid j}^{\prime}\left(\varphi_{1}^{N_{1}}\right)>E_{i \mid j}^{\prime}\left(\varphi_{1}\right)-\varepsilon
$$

The set of all achievable vectors is defined by $\mathcal{R}^{\prime}$.

Let us define a region $\mathcal{E}^{\prime}$ in the error-exponent space as follows:

$$
\mathcal{E}^{\prime} \triangleq\left\{\mathbf{E}^{\prime}: \forall Q, \exists i, \min _{s: s \in \mathcal{D}_{j}} \mathrm{D}\left(Q \| P_{s}\right)>E_{i \mid j}^{\prime}\left(\varphi_{1}\right), \quad j \neq i, \quad i, j=1,2\right\}
$$

So we have

$$
\mathcal{E}^{\prime}=\left\{\mathbf{E}^{\prime}: \forall Q, \min _{s: s \in \mathcal{D}_{1}} \mathrm{D}\left(Q \| P_{s}\right)>E_{2 \mid 1}^{\prime}\left(\varphi_{1}\right), \text { or } \min _{s: s \in \mathcal{D}_{2}} \mathrm{D}\left(Q \| P_{s}\right)>E_{1 \mid 2}^{\prime}\left(\varphi_{1}\right)\right\}
$$

Theorem 4.2 The following inclusion take place $\mathcal{E}^{\prime} \subset \mathcal{R}^{\prime}$. Conversely if $\mathbf{E}^{\prime} \in \mathcal{R}^{\prime}$, then for any $\delta>0, \mathbf{E}_{\delta}^{\prime} \in \mathcal{E}^{\prime}$, where $\mathbf{E}_{\delta}^{\prime}=\left\{E_{i \mid j}^{\prime}\left(\varphi_{1}\right)-\delta\right\}$. 
Proof. If $\mathbf{E}^{\prime} \in \mathcal{E}^{\prime}$, then

$$
\begin{aligned}
\alpha_{2 \mid 1}^{\prime}\left(\varphi_{1}^{N_{1}}\right)= & \max _{s: s \in \mathcal{D}_{1}} P_{s}^{N_{1}}\left(\mathcal{A}_{2}^{N_{1}}\right)=\max _{s: s \in \mathcal{D}_{1}} \sum_{\mathrm{x}_{1} \in \mathcal{A}_{2}^{N_{1}}} P_{s}^{N_{1}}\left(\mathrm{x}_{1}\right) \\
= & \max _{s: s \in \mathcal{D}_{1}} \sum_{Q_{\mathrm{x}_{1}}: \mathcal{T}_{Q_{\mathrm{x}_{1}}}^{N_{1}} \subset \mathcal{A}_{2}^{N_{1}}} P_{s}^{N_{1}}\left(\mathrm{x}_{1} \in \mathcal{T}_{Q_{\mathrm{x}_{1}}}^{N_{1}}\right) \\
= & \max _{s: s \in \mathcal{D}_{1}} \sum_{Q_{\mathrm{x}_{1}}: \mathcal{T}_{Q_{\mathrm{x}_{1}}}^{N_{1}} \subset \mathcal{A}_{2}^{N_{1}}}\left|\mathcal{T}_{Q_{\mathrm{x}_{1}}}^{N_{1}}\right| \exp \left\{-N_{1}\left\{\mathrm{H}\left(Q_{\mathrm{x}_{1}}\right)+\mathrm{D}\left(Q_{\mathrm{x}_{1}} \| P_{s}\right)\right\}\right\} \\
\leq & \max _{s: s \in \mathcal{D}_{1}} \sum_{Q_{\mathrm{x}_{1}}: \mathcal{T}_{Q_{\mathrm{x}_{1}}}^{N_{1}} \subset \mathcal{A}_{2}^{N_{1}}} \exp \left\{N_{1} \mathrm{H}\left(Q_{\mathrm{x}_{1}}\right)\right\} \exp \left\{-N_{1}\left\{\mathrm{H}\left(Q_{\mathrm{x}_{1}}\right)+\mathrm{D}\left(Q_{\mathrm{x}_{1}} \| P_{r}\right)\right\}\right\} \\
= & \max _{s: s \in \mathcal{D}_{1}} \sum_{Q_{\mathrm{x}_{1}}: \mathcal{T}_{Q_{\mathrm{x}_{1}}}^{N_{1}} \subset \mathcal{A}_{2}^{N_{1}}} \exp \left\{-N_{1} \mathrm{D}\left(Q_{\mathrm{x}_{1}} \| P_{s}\right)\right\} \\
= & \left.\sum_{Q_{\mathrm{x}_{1}}: \mathcal{T}_{Q_{\mathrm{x}_{1}}^{N_{1}} \subset \mathcal{A}_{2}} \exp \left\{-N_{1}^{N_{1}}\right.} \min _{s: s \in \mathcal{D}_{1}} \mathrm{D}\left(Q_{\mathrm{x}_{1}} \| P_{s}\right)\right\} \\
\leq & \sum_{Q_{\mathrm{x}_{1}}: \mathcal{T}_{Q_{\mathrm{x}_{1}}}^{N_{1}} \subset \mathcal{A}_{2}^{N_{1}}} \exp \left\{-N_{1} E_{2 \mid 1}^{\prime}\right\} \\
& <\left(N_{1}+1\right)^{|\mathcal{X}|} \exp \left\{-N_{1} E_{2 \mid 1}^{\prime}\right\} \\
= & \exp \left\{-N_{1} E_{2 \mid 1}^{\prime}+|\mathcal{X}| \log \left(N_{1}+1\right)\right\} \\
= & \exp \left\{-N_{1}\left\{E_{2 \mid 1}^{\prime}-\varepsilon\right\}\right\} .
\end{aligned}
$$

So we have

$$
-\frac{1}{N_{1}} \log \alpha_{2 \mid 1}^{\prime}\left(\varphi_{1}^{N_{1}}\right)>E_{2 \mid 1}^{\prime}-\varepsilon
$$

and similarly we get

$$
-\frac{1}{N_{1}} \log \alpha_{1 \mid 2}^{\prime}\left(\varphi_{1}^{N_{1}}\right)>E_{1 \mid 2}^{\prime}-\varepsilon
$$

Therefore $\mathbf{E}^{\prime}=\left\{E_{2 \mid 1}^{\prime}, E_{1 \mid 2}^{\prime}\right\}$ is achievable and $\mathbf{E}^{\prime} \in \mathcal{R}^{\prime}$, so the first part of Theorem accomplished.

Conversely in the second part of Theorem if $\mathbf{E}^{\prime} \in \mathcal{R}^{\prime}$ then for any $N_{1}>N_{1}(\varepsilon)$ we have:

$$
-\frac{1}{N_{1}} \log \alpha_{2 \mid 1}^{\prime}\left(\varphi_{1}^{N_{1}}\right)>E_{2 \mid 1}^{\prime}-\varepsilon \text { and }-\frac{1}{N_{1}} \log \alpha_{1 \mid 2}^{\prime}\left(\varphi_{1}^{N_{1}}\right)>E_{1 \mid 2}^{\prime}-\varepsilon
$$

If $\mathbf{E}_{\delta}^{\prime} \notin \mathcal{E}^{\prime}$ then there exist a distribution $Q$ such that:

$$
\min _{s: s \in \mathcal{D}_{1}} \mathrm{D}\left(Q \| P_{s}\right) \leq E_{2 \mid 1}^{\prime}-\delta, \text { and } \min _{s: s \in \mathcal{D}_{2}} \mathrm{D}\left(Q \| P_{s}\right) \leq E_{1 \mid 2}^{\prime}-\delta .
$$

Using the continuity properties of $\mathrm{D}\left(Q \| P_{s}\right)$ for a fixed $s$ and $\mathcal{P}^{N_{1}}(\mathcal{X})$ is dense in all PDs, we can find for large enough $N_{1}$ and $Q_{\mathrm{x}_{1}} \in \mathcal{P}^{N_{1}}(\mathcal{X})$,

$$
\mathrm{D}\left(Q_{\mathrm{x}_{1}} \| P_{s}\right) \leq \mathrm{D}\left(Q \| P_{s}\right)+\delta / 2
$$

Then it is clear that for all $s: s \in \mathcal{D}_{1}$, we receive to

$$
\begin{aligned}
\min _{s: s \in \mathcal{D}_{1}} \mathrm{D}\left(Q_{\mathrm{x}_{1}} \| P_{s}\right) & \leq \min _{s: s \in \mathcal{D}_{1}} \mathrm{D}\left(Q \| P_{s}\right)+\delta / 2 \\
& \leq E_{2 \mid 1}^{\prime}-\delta+\delta / 2
\end{aligned}
$$

and we receive to $\min _{s: s \in \mathcal{D}_{1}} \mathrm{D}\left(Q_{\mathrm{x}_{1}} \| P_{s}\right) \leq E_{2 \mid 1}^{\prime}-\delta / 2<E_{2 \mid 1}^{\prime}$, and consequently we get

$$
\mathcal{A}_{2}^{\left(N_{1}\right)}=\bigcup_{Q_{\mathbf{x}_{1}}: \min _{s: s \in \mathcal{D}_{1}} \mathrm{D}\left(Q_{\mathbf{x}_{1}} \| P_{s}\right)>E_{2 \mid 1}^{\prime}} \mathcal{T}_{Q_{\mathbf{x}_{1}}}^{N_{1}}=\emptyset,
$$

that it contradict to Definition 4.1 and there is $\mathbf{E}_{\delta}^{\prime} \in \mathcal{E}^{\prime}$ and proof of Theorem is completed. 


\section{Achievable region at the second stage of two-stage test}

At the second stage of decision making the test is $\varphi_{2}^{N_{2}}\left(\mathrm{x}_{2} \mid \varphi_{1}^{N_{1}}\left(\mathrm{x}_{1}\right)=i\right), i=1,2$, if the first (or the second) family of PDs is accepted, then it can be defined by partitioning the sample space $\mathcal{X}^{N_{2}}$ to $R$ (or $S-R$ ) disjoint subsets $\mathcal{B}_{s}^{\left(N_{2}\right)}, s \in \mathcal{D}_{1}\left(\right.$ or $\left.\mathcal{B}_{s}^{\left(N_{2}\right)}, s \in \mathcal{D}_{2}\right)$. The set $\mathcal{B}_{s}^{\left(N_{2}\right)}$ consists of all vectors $\mathrm{x}_{2}$ for which $s$-th PD is adopted. So if the $i$-th family of PDs is accepted, then

$$
\begin{aligned}
& \mathcal{B}_{s}^{\left(N_{2}\right)} \triangleq\left\{\mathrm{x}_{2}: \varphi_{2}^{N_{2}}\left(\mathrm{x}_{2} \mid \varphi_{1}^{N_{1}}\left(\mathrm{x}_{1}\right)=i\right)=s\right\}, \quad s \in \mathcal{D}_{i}, \quad i=1,2, \\
& \bigcup_{s \in \mathcal{D}_{i}} \mathcal{B}_{s}^{\left(N_{2}\right)}=\mathcal{X}^{N_{2}}, \quad \mathcal{B}_{s}^{\left(N_{2}\right)} \cap \mathcal{B}_{l}^{\left(N_{2}\right)}=\emptyset, \quad l \neq s \in \mathcal{D}_{i}, \quad i=1,2 .
\end{aligned}
$$

Let $\alpha_{l \mid k}^{\prime \prime}\left(\varphi_{2}^{N_{2}}\right)$ be the probability of the erroneous acceptance of PD $P_{l}$ at the second stage of test provided $P_{k}$ is true:

$$
\alpha_{l \mid k}^{\prime \prime}\left(\varphi_{2}^{N_{2}}\right) \triangleq P_{k}^{N_{2}}\left(\mathcal{B}_{l}^{\left(N_{2}\right)}\right), \quad l \in \mathcal{D}_{i}, \quad l \neq k, \quad i=1,2
$$

The probability to reject $P_{k}$, when it is true, is

$$
\alpha_{k \mid k}^{\prime \prime}\left(\varphi_{2}^{N_{2}}\right) \triangleq P_{k}^{N_{2}}\left(\overline{\mathcal{B}}_{k}^{\left(N_{2}\right)}\right)=\sum_{l \neq k} \alpha_{l \mid k}^{\prime \prime}\left(\varphi_{2}^{N_{2}}\right), l \in \mathcal{D}_{i}, i=1,2
$$

For the infinite sequences of tests $\varphi_{2}$, corresponding reliabilities are defined as

$$
E_{l \mid k}^{\prime \prime}\left(\varphi_{2}\right) \triangleq \liminf _{N_{2} \rightarrow \infty}\left\{-\frac{1}{N_{2}} \log \alpha_{l \mid k}^{\prime \prime}\left(\varphi_{2}^{N_{2}}\right)\right\}, \quad l \in \mathcal{D}_{i}, \quad i=1,2 .
$$

It follows from (1) and (2) that

$$
E_{k \mid k}^{\prime \prime}\left(\varphi_{2}\right)=\min _{l \neq k} E_{l \mid k}^{\prime \prime}\left(\varphi_{2}\right) .
$$

We denote by $\mathbf{E}^{\prime \prime}=\left\{E_{l \mid k}^{\prime \prime}, l \neq k, l, k=\overline{1, S}\right\}$ the vector, element of which correspond to the set of error exponents $-\frac{1}{N_{2}} \log \left\{P_{k}^{N_{2}}\left(\mathcal{B}_{l}^{\left(N_{2}\right)}\right)\right\}$.

Similar to Definition 3.1. the set of error exponents indicated by vector $\mathbf{E}^{\prime \prime}$ is called achievable if for all $\varepsilon>0$ and large enough $N_{2}$ there exists a decision schemes $\mathcal{B}_{l}^{\left(N_{2}\right)}, l \in \mathcal{D}_{i}, i=1,2$, satisfying for $l, k=\overline{1, S}, l \neq k$ the following conditions:

$$
-\frac{1}{N_{2}} \log \alpha_{l \mid k}^{\prime \prime}\left(\varphi_{2}^{N_{2}}\right)>E_{l \mid k}^{\prime \prime}\left(\varphi_{2}\right)-\varepsilon
$$

The set of all achievable vectors is defined by $\mathcal{R}^{\prime \prime}$.

Let us define a region $\mathcal{E}^{\prime \prime}$ in the error-exponent space as follows:

$$
\mathcal{E}^{\prime \prime} \triangleq\left\{\mathbf{E}^{\prime \prime}: \forall Q, \exists l, \mathrm{D}\left(Q \| P_{k}\right)>E_{l \mid k}^{\prime \prime}\left(\varphi_{2}\right), \quad k \neq l, \quad l, k=\overline{1, S}\right\} .
$$

Theorem 5.1 [11] The following inclusion take place $\mathcal{E}^{\prime \prime} \subset \mathcal{R}^{\prime \prime}$. Conversely if $\mathbf{E}^{\prime \prime} \in \mathcal{R}^{\prime \prime}$, then for any $\delta>0$, $\mathbf{E}_{\delta}^{\prime \prime} \in \mathcal{E}^{\prime \prime}$, where $\mathbf{E}_{\delta}^{\prime \prime}=\left\{E_{l \mid k}^{\prime \prime}\left(\varphi_{2}\right)-\delta\right\}$.

\section{Achievable region at the two-stage test}

The test $\Phi^{N}=\left(\varphi_{1}^{N_{1}}, \varphi_{2}^{N_{2}}\right)$ is composed by a pair of tests $\varphi_{1}^{N_{1}}$ and $\varphi_{2}^{N_{2}}$. In the two-stage decision making, if at the first stage of test, the $i$-th family of PDs is accepted then the test $\Phi^{N}$, can be defined by partitioning the sample space $\mathcal{X}^{N}$ to $S$ disjoint subsets as follows:

$$
\begin{gathered}
\mathcal{C}_{s}^{(N)} \triangleq \mathcal{A}_{i}^{\left(N_{1}\right)} \times \mathcal{B}_{s}^{\left(N_{2}\right)}, \quad s \in \mathcal{D}_{i}, \quad i=1,2 \\
\mathrm{x}=\left(\mathrm{x}_{1}, \mathrm{x}_{2}\right) \in \mathcal{C}_{s}^{(N)}: \quad \mathrm{x}_{1} \in \mathcal{A}_{i}^{\left(N_{1}\right)}, \quad \mathrm{x}_{2} \in \mathcal{B}_{s}^{\left(N_{2}\right)}, s \in \mathcal{D}_{i}, \quad i=1,2 .
\end{gathered}
$$


and the set $\mathcal{C}_{s}^{(N)}$ consists of all vectors $\mathrm{x}$ for which in the two-stage test $s$-th PD is adopted and $\bigcup_{s=1}^{S} \mathcal{C}_{s}^{(N)}=\mathcal{X}^{N}$.

Let $\alpha_{l \mid k}^{\prime \prime \prime}$ be the probability of the erroneous acceptance of $\mathrm{PD} P_{l}$ at the two-stage test, provided $P_{k}$ is true:

$$
\alpha_{l \mid k}^{\prime \prime \prime}\left(\Phi^{N}\right) \triangleq P_{k}^{N}\left(\mathcal{C}_{l}^{(N)}\right), \quad l, k=\overline{1, S}, \quad l \neq k
$$

And the probability to reject $P_{k}$ at the two-stage test, when it is true, is

$$
\alpha_{k \mid k}^{\prime \prime \prime}\left(\Phi^{N}\right) \triangleq P_{k}^{N}\left(\overline{\mathcal{C}}_{k}^{(N)}\right)=\sum_{l \neq k} \alpha_{l \mid k}^{\prime \prime \prime}\left(\Phi^{N}\right), \quad l, k=\overline{1, S}
$$

The reliabilities of the infinite sequences of tests $\Phi=\left(\varphi_{1}, \varphi_{2}\right)$ are defined as

$$
E_{l \mid k}^{\prime \prime \prime}(\Phi) \triangleq \liminf _{N \rightarrow \infty}\left\{-\frac{1}{N} \log \alpha_{l \mid k}^{\prime \prime \prime}\left(\Phi^{N}\right)\right\}, \quad l, k=\overline{1, S} .
$$

We denote by $\mathbf{E}^{\prime \prime \prime}=\left\{E_{l \mid k}^{\prime \prime \prime}, l \neq k, l, k=\overline{1, S}\right\}$ the vector, element of which correspond to the set of error exponents $-\frac{1}{N} \log \left\{P_{k}^{N}\left(\mathcal{C}_{l}^{(N)}\right)\right\}$.

Definition 6.1 [11] The set of error exponents indicated by vector $\mathbf{E}^{\prime \prime \prime}$ is called achievable if for all $\varepsilon>0$ and large enough $N$ there exists a decision schemes $\mathcal{C}_{l}^{(N)}, l=\overline{1, S}$ satisfying for $l, k=\overline{1, S}, l \neq k$ the following conditions:

$$
-\frac{1}{N} \log \alpha_{l \mid k}^{\prime \prime \prime}\left(\Phi^{N}\right)>E_{l \mid k}^{\prime \prime \prime}(\Phi)-\varepsilon
$$

The set of all achievable vectors is defined by $\mathcal{R}^{\prime \prime \prime}$.

Let us define a region $\mathcal{E}^{\prime \prime \prime}$ in the error-exponent space as follows:

$$
\mathcal{E}^{\prime \prime \prime} \triangleq\left\{\mathbf{E}^{\prime \prime \prime}: \forall Q, \exists l, \mathrm{D}\left(Q \| P_{k}\right)>E_{l \mid k}^{\prime \prime \prime}(\Phi), \quad k \neq l, \quad l, k=\overline{1, S}\right\} .
$$

Theorem 6.2 The following inclusion take place $\mathcal{E}^{\prime \prime \prime} \subset \mathcal{R}^{\prime \prime \prime}$. Conversely if $\mathbf{E}^{\prime \prime \prime} \in \mathcal{R}^{\prime \prime \prime}$, then for any $\delta>0$, $\mathbf{E}_{\delta}^{\prime \prime \prime} \in \mathcal{E}^{\prime \prime \prime}$, where $\mathbf{E}_{\delta}^{\prime \prime \prime}=\left\{E_{l \mid k}^{\prime \prime \prime}(\Phi)-\delta\right\}$.

Proof. If $\mathbf{E}^{\prime \prime \prime} \in \mathcal{E}^{\prime \prime \prime}$ then for any type $Q$ there exist at least one $l$ such that $\mathrm{D}\left(Q \| P_{k}\right)>E_{l \mid k}^{\prime \prime \prime}(\Phi)$ for all $k \neq l$. If there ara multiple such $l$, select one arbitrarily and assign the all type class $\mathcal{T}_{Q}^{N}$ to $\mathcal{C}_{l}^{(N)}$. The result of probability of error corresponding satisfies the following chain of inequalities:

$$
\begin{aligned}
P_{k}^{N}\left(C_{l}^{(N)}\right)= & \sum_{\mathrm{x} \in C_{l}^{(N)}} P_{k}^{N}(\mathrm{x}) \\
= & \sum_{Q: \mathcal{T}_{Q}^{N} \subset C_{l}^{(N)}}\left|\mathcal{T}_{Q}^{N}\right| \exp \left\{-N\left\{\mathrm{H}(Q)+\mathrm{D}\left(Q \| P_{k}\right)\right\}\right\} \\
\leq & \sum_{Q: \mathcal{T}_{Q}^{N} \subset C_{l}^{(N)}} \exp \left\{-N \mathrm{D}\left(Q \| P_{k}\right)\right\} \\
< & (N+1)^{|\mathcal{X}|} \exp \left\{-N \mathrm{D}\left(Q \| P_{k}\right)\right\} \\
\leq & \exp \left\{-N\left\{E_{l \mid k}^{\prime \prime \prime}-\varepsilon\right\}\right\}
\end{aligned}
$$

So we have

$$
-\frac{1}{N} \log \left\{\alpha_{l \mid k}^{\prime \prime \prime}\left(\Phi^{N}\right)\right\}=-\frac{1}{N} \log \left\{P_{k}^{N}\left(C_{l}^{(N)}\right)\right\}>E_{l \mid k}^{\prime \prime \prime}(\Phi)-\varepsilon .
$$

Consequently the first part of Theorem is proved.

Conversely in the second part of Theorem if $\mathbf{E}^{\prime \prime \prime} \in \mathcal{R}^{\prime \prime \prime}$ then for every $\varepsilon>0$, there exists some sequence of decision schemes $C_{l}^{(N)}, l=\overline{1, S}$ and some number $N>N(\varepsilon)$ such that for all $l \neq k$

$$
-\frac{1}{N} \log \left\{P_{k}^{N}\left(\mathcal{C}_{l}^{(N)}\right)\right\}>E_{l \mid k}^{\prime \prime \prime}(\Phi)-\varepsilon .
$$


If $\mathbf{E}_{\delta}^{\prime \prime \prime} \notin \mathcal{E}^{\prime \prime \prime}$, then there exists a distribution $Q$ and $\delta>0$ such that

$$
\exists l: \mathrm{D}\left(Q \| P_{k}\right) \leq E_{l \mid k}^{\prime \prime \prime}(\Phi)-\delta, \quad k \neq l, \quad l, k=\overline{1, S} .
$$

Using the continuity properties of $\mathrm{D}\left(Q \| P_{k}\right)$ for a fixed $k$ and $\mathcal{P}^{N}(\mathcal{X})$ is dense in all PDs, we can find for large enough $N$ and $Q_{\mathrm{x}} \in \mathcal{P}^{N}(\mathcal{X})$,

$$
\begin{aligned}
\mathrm{D}\left(Q_{\mathrm{x}} \| P_{k}\right) & \leq \mathrm{D}\left(Q \| P_{k}\right)+\delta / 2 \\
& \leq E_{l \mid k}^{\prime \prime \prime}(\Phi)-\delta / 2 .
\end{aligned}
$$

The corresponding result of error probabilities satisfy the following chain of inequalities:

$$
\begin{aligned}
P_{k}^{N}\left(C_{l}^{(N)}\right)= & \sum_{\mathrm{x} \in C_{l}^{(N)}} P_{k}^{N}(\mathrm{x}) \\
& =\sum_{Q_{\mathrm{x}}: \mathcal{T}_{Q_{\mathrm{x}}}^{N} \subset C_{l}^{(N)}}\left|\mathcal{T}_{Q_{\mathrm{x}}}^{N}\right| \exp \left\{-N\left\{\mathrm{H}\left(Q_{\mathrm{x}}\right)+\mathrm{D}\left(Q_{\mathrm{x}} \| P_{k}\right)\right\}\right\} \\
& \geq(N+1)^{-|\mathcal{X}|} \exp \left\{-N \mathrm{D}\left(Q_{\mathrm{x}} \| P_{k}\right)\right\} \\
& \geq(N+1)^{-|\mathcal{X}|} \exp \left\{-N\left\{E_{l \mid k}^{\prime \prime \prime}(\Phi)-\delta / 2\right\}\right\} \\
& =\exp \left\{-N\left\{E_{l \mid k}^{\prime \prime \prime}(\Phi)-\delta^{*}\right\}\right\}
\end{aligned}
$$

So we have

$$
-\frac{1}{N} \log \left\{\alpha_{l \mid k}^{\prime \prime \prime}\left(\Phi^{N}\right)\right\}=-\frac{1}{N} \log \left\{P_{k}^{N}\left(C_{l}^{(N)}\right)\right\} \leq E_{l \mid k}^{\prime \prime \prime}(\Phi)-\delta^{*} .
$$

But this last inequality contradicts with Definition 6.1. and therefore $\mathbf{E}^{\prime \prime \prime} \notin \mathcal{R}^{\prime \prime \prime}$. Consequently if $\mathbf{E}^{\prime \prime \prime} \in \mathcal{R}^{\prime \prime \prime}$, then $\mathbf{E}_{\delta}^{\prime \prime \prime} \in \mathcal{E}^{\prime \prime \prime}$ and the converse part of Theorem is accomplished.

\section{Conclusion}

The achievable region of reliabilities in multiple hypothesis testing problem for an object that follows a PD among one of the pair of disjoint families of PDs is exposed . In the two-stage test, at the first stage of test $\varphi_{1}$, an achievable region of reliabilities $\mathcal{R}^{\prime}$ and at the second stage of test $\varphi_{2}$ an achievable region of reliabilities $\mathcal{R}^{\prime \prime}$ are investigated and consequently at the two-stage test $\Phi=\left(\varphi_{1}, \varphi_{2}\right)$ is shown by Theorem 6.2. that there exists an achievable region of reliabilities $\mathcal{R}^{\prime \prime \prime}$ and the defined region for the vectors of reliabilities of the two-stage test characterizes the set of all achievable vectors. Also advantages of procedures of the two-stage LAO testing was revealed in [8].

\section{Acknowledgments}

This work was supported by Ahvaz branch, Islamic Azad University, grant number 51063910508006 .

\section{References}

[1] R. Ahlswede and E.A. Haroutunian, On statistical hypotheses optimal testing and identification, Lecture Notes in Computer Science, General Theory of Information Transfer and Combinatorics, Springer, 4123 (2006), $462-478$.

[2] T.M. Cover and J.A. Tomas, Element of information theory, (Second Edition), Wiley, New York, 2006.

[3] I. Csiszár and J. Körner, Information theory: coding theorems for discrete memoryless systems, Academic Press, New York, 1981.

[4] I. Csiszár and P.C. Shields, Information theory and statistics: a tutorial, Foundations and Trends in Communications and Information Theory, 1:4 (2004), 417-528.

[5] S. Dudua and M.J. Van Der Laon, Multiple testing procedures with application to genomics, Springer, 2008.

[6] E.A. Haroutunian, Logarithmically asymptotically optimal testing of multiple statistical hypotheses, Problems of Control and Information Theory, 19 (1990), 413-421. 
[7] E.A. Haroutunian, M.E. Haroutunian and A.N. Haroutunyan, Reliability criteria in information theory and in statistical hypothesis testing, Foundations and Trends in Communications and Information Theory, 4:2-3 (2008), 97-263.

[8] E.A. Haroutunian, P.M. Hakobyan and F. Hormozi-nejad, On Two-stage LAO Testing of Multiple Hypotheses for a Pair of Families of Probability Distributions, Journal of Statistical and Econometric Methods, 2:2 (2013), 127-156.

[9] W. Hoefding, Asymptotically optimal tests for multinomial distributions, Annals of Mathematical Statistics, 36 (1965), 369-401.

[10] E.L. Lehman and J.P. Romano, Testing statistical hypotheses, (2nd Edition), Springer, 2005.

[11] E. Tuncel, On error exponents in hypothesis testing, IEEE Trans. Inf. Theory, 51:8 (2005), 2945-2950.

[12] G. Tusnady, On asymptotically optimal tests, Annals of Statistics, 5:2 (1977), 385-393.

[13] A.O. Yessayan, E.A. Haroutunian and P.M. Hakobyan, Error probability exponents and achievable region in testing of many hypotheses for two independent objects, Mathematical Problems of Computers Science, 35 (2011), 33-36. 